\title{
Aspectos de sostenibilidad de productos no maderables forestales con uso curativo en el oeste de Pará, Brasi**
}

\author{
Aspects of Sustainability of Non-timber Forest Products \\ Used for Health in Western Pará, Brazil
}

\section{Aspects de la durabilité de produits forestiers non ligneuses a usage curatif dans le ovest de Pará, Brésil}

\section{Thomas May**}

Recibido: 2015-04-20 // Aprobado: 2015-07-10 // Disponible en línea: 2016-01-30

Cómo citar este artículo: May, T. (2016). Aspectos de sostenibilidad de productos no maderables forestales con uso curativo en el oeste de Pará, Brasil. Ambiente y Desarrollo, 20(38), 69-84. http://dx.doi.org/10.11144/Javeriana.ayd20-38.aspm

doi:10.11144/Javeriana.ayd20-38.aspm

\begin{abstract}
Resumen
Se analiza la sostenibilidad de los doce productos no maderables forestales con fines curativos más vendidos en el mercado local en el oeste del estado de Pará, Brasil, con base en observaciones e informaciones bibliográficas, tomando como hilo conductor factores de origen externo versus factores internos, y la disponibilidad de los recursos naturales versus aspectos económicos y sociales. Se concluye que 1) la destrucción y degradación de los bosques tiene el potencial de afectar negativamente a los recursos, si bien a corto plazo contribuye a un aumento de la oferta de ciertos productos; 2 ) los precios y los volúmenes de venta parecen ser más estables en el mercado local que en mercados más distantes; 3) las características biológicas de algunas especies indican que existe un potencial de uso sostenible, mientras que en otras se vislumbra un impacto negativo en el recurso; y 4) en varias especies la plantación a escala local es una estrategia prometedora para enfrentar la reducción de la disponibilidad del recurso, aumentando la eficiencia del trabajo de recolección y acopio.
\end{abstract}

Palabras clave: productos no maderables de los bosques; Amazonía brasileña; sostenibilidad; factores externos e internos; disponibilidad de los recursos/factores económicos y sociales

\footnotetext{
* $\quad$ Esta investigación se deriva de la actividad del autor como cooperante del Servicio Alemán de Cooperación Social y Técnica con la flona Tapajós en Santarém/Pará, de 2005 a 2007, y en la Oficina Regional del Servicio Alemán en Santarém, de 2007 a 2008.

** Biólogo, doctor en Ciencias (Geografía), experto integrado del cim y docente investigador en la Universidad Estatal Amazónica (UEA), vía a Napo km 2 1/2, paso lateral. Puyo, Ecuador. Correos electrónicos: may_ gutierreztr@yahoo.es, tmay@uea.edu.ec.
} 


\begin{abstract}
Sustainability of the twelve curative non timber products of amazonian forests which are most sold on the local market in the western part of the Pará state (Brazil) is analyzed, based on field observations and bibliographic informations, taking as a guideline the factors which have an impact, of external versus internal origin, and of impact on the disponibility of the ressources versus of impact on economic and social aspects. It is concluded that 1) forest destruction and degradation makes expect negative impacts on the NTFP, even if at the short term the offer of certain products may rise, 2) prices and selling volumes on local markets seem to be more stable than on distant markets, 3) biological traits of some of the species indicate that there is a potential of sustainable use, whereas in others there could be a negative impact on the ressoure, and 4) in some species plantation at a local scale is a promisory adaptative strategy to face the depletion of the ressource, by increasing the efficiency of local labour.
\end{abstract}

Keywords: non timber forest products; Brazilian Amazon; sustainability; external/ internal factors; ressource disponibility/economic and social factors

\title{
Résumé
}

La durabilité de douce produits non ligneuses de la foret a usage curatif qui sont les plus vendus dans le ouest de l'état brésilien du Pará est analysé, basé dans des observations et des informations bibliographiques, en s'orientant dans des facteurs d'influence, d'origine externe face à origine interne, et d'impact sur la disponibilité des ressources face à l'impact sur des aspects économiques et sociaux. Les conclusions sont : 1) a moyen et long terme, il est prévisible un impact négatif de la destruction et dégradation des forêts, bien qu' à court terme l'offre de certains produits augmente, 2) il parait que les prix et les quantités vendus sont plus stables sur le marché local que sur les marchés plus distantes, 3) les caractéristiques biologiques indiquent que dans certaines espèces il y a un potentiel d' utilisation durable, tandis qu'en d'autres il pourrait avoir des difficultés, et 4) pour quelques espèces la plantation a échelle locale est une possible stratégie pour faire face à la réduction de la disponibilité des ressources, en augmentant l'efficience du travail.

Mots clés : produits non ligneuses de la foret; Amazonie brésilienne; durabilité; facteurs d'origine externe et interne; disponibilité des ressources/facteurs économiques et sociaux 


\section{Introducción}

La extracción de productos no maderables de los bosques ha tenido importantes impactos económicos y sociales en la región amazónica. Entre los ejemplos más conocidos están los casos de la castaña de Brasil (Bertholletia excelsa) y del caucho (Hevea brasiliensis), productos con venta en los mercados internacionales (Zuidema, 2003; Carrasco, 2005). En el caso del caucho, cabe señalar que el producto, después de un auge durante varias décadas a finales del siglo XIX e inicios del siglo XX, sufrió una notable competencia de parte del caucho producido en plantaciones, lo que redujo mucho el volumen del producto proveniente de fuentes extractivas, y sus perspectivas de venta y precios cambiaban según coyunturas nacionales e internacionales, como las políticas del gobierno central de Brasil con respecto a la Amazonía y la Segunda Guerra Mundial (Weinstein, 1997; Almeida, 2004).

A pesar de su gran importancia, los productos forestales no maderables (PFNM) han recibido atención a nivel mundial y en el ámbito científico solo a partir de la década de 1980 (López Camacho, 2008). Existe una gran variedad de definiciones de los PFNM que tienen en común considerarlos productos del bosque y no incluir la madera. Si con la extracción de los PFNM los árboles generalmente se quedan en pie y no se retiran del ecosistema, es razonable suponer que el impacto de su uso en la estructura del bosque, en los flujos de energía y ciclos de nutrientes, así como en la biodiversidad, debe ser sensiblemente menor que en el caso del aprovechamiento de la madera. Por el otro lado, quienes participan en las cadenas de valor de los PFNM tienen un interés económico en que se mantengan los bosques para que la producción tenga continuidad, y es de esperar que sean aliados en la conservación de la biodiversidad, a menos que se trate de grandes inversionistas, suficientemente flexibles para retirar su capital e invertirlo en otros sectores, cuando las cadenas de valor de los PFNM se hacen menos rentables, como ha sucedido en el caso del caucho (Almeida, 2004).

Existen diversos factores que pueden favorecer o comprometer la sostenibilidad de los sistemas de uso y manejo de los recursos naturales. De acuerdo con Young y Burton (1992), a nivel de recursos o ecosistemas específicos, el concepto de sostenibilidad se relaciona con lo que se describe con el término de resiliencia (resilience), que se refiere a la capacidad de un sistema - ecológico u otro- de mantener las relaciones entre sus componentes ante impactos externos (Holling, 1973). Por el otro lado, el concepto de sostenibilidad no se limita a la resiliencia de los ecosistemas involucrados, sino que implica que existe una capacidad de producción de bienes o servicios que perdura por un tiempo prolongado, lo que significa que esta capacidad no se desgaste (Young y Burton, 1992).

El sistema de uso es más amplio que el ecosistema que produce los bienes y servicios, e incluye componentes técnicos, económicos, sociales y culturales. Por lo tanto, al evaluar la sostenibilidad de los sistemas de uso, es necesario considerar también estos aspectos (Masera et al., 2000; De Groot y Steg, 2009; Arnés Prieto, 2011), con la misma importancia que el subsistema ecológico, e integrar los diferentes componentes en una visión conjunta (Carpenter et al., 2009). Dada la escasa disponibilidad de datos, en el caso presente estudiar todos los aspectos enunciados resultaría altamente especulativo. Por lo tanto, para sistematizar los aspectos de sostenibilidad de determinados productos no maderables del bosque, se consideran dos dimensiones: la primera se refiere al tipo de factores: los ecológicos, es decir, relacionados con la capacidad del ecosistema de generar los productos y servicios a corto y a largo plazo, y los sociales en un sentido amplio, relacionados con la recolección, transformación, calidad del producto, venta y bienestar social (1). La otra dimensión se refiere al origen de los factores que pueden afectar la sostenibilidad, que puede ser interno o externo al sistema local de uso (2).

Cada producto tiene su propio sistema de uso, por lo que en el presente trabajo, los aspectos de sostenibilidad se van analizando por productos. Claro está que existen otros aspectos importantes de la sostenibilidad (Becker y Ostrom, 1995; Masera et al., 2000), relacionados con el grado organizativo de los grupos de productores, con las instituciones sociales en general, infraestructura, así como la satisfacción con la forma de vida y con el trabajo, que no se van a analizar aquí. El objetivo del presente estudio es identificar y comparar los principales aspectos internos y externos, ecológicos y sociales (en 
el sentido más amplio), que favorecen o dificultan la sostenibilidad del sistema de uso de los distintos productos. Debido a la escasa disponibilidad de informaciones relevantes, hay que considerar las conclusiones como preliminares.

\section{Zona de estudio y metodología}

La ciudad de Santarém es el centro administrativo, político y comercial del oeste del estado brasileño de Pará, y tenía aproximadamente 180000 habitantes en el momento del levantamiento de los datos del estudio (2006-2008). En los alrededores, hasta la segunda mitad del siglo xx los asentamientos rurales de caboclos se habían concentrado en las orillas de los ríos. Posteriormente, con la construcción de ejes viales como la BR 163 y la Transamazónica, se observó un avance de la frontera agrícola, proceso que se intensificó desde la última década del siglo xx (Bacha y Rodríguez, 2005), junto con actividades de explotación maderera. El Mercadão 2000 de Santarém es un mercado público que fue establecido por la municipalidad de esa ciudad a principios de la década de 2000, como un espacio de comercialización para productores familiares y pequeńos comerciantes locales. Además de frutas, hortalizas y alimentos de base, son ofertados varios PFNM, la mayoría para fines curativos. En la población tradicional de las comunidades en las orillas de los ríos, existe una tradición de este tipo de usos de productos forestales no maderables (Araujo, 2002).

De enero de 2006 hasta mayo de 2008 el autor realizó visitas del Mercadão 2000 de Santarém, registrando los productos naturales ofertados por los comerciantes. Las visitas se efectuaron con un ritmo mensual, o con mayor frecuencia, a excepción de julio de 2007 cuando no se hizo ninguna visita. El total de visitas fue de 34. Para los análisis posteriores se tomaron en cuenta los productos de plantas que fueron encontrados en las mesas de los comerciantes en por lo menos 31 de las 34 visitas, lo que corresponde a más de $90 \%$ de todas las visitas realizadas. En cada visita se registraron los precios de los productos y se contaron los números de botellas ofertadas - generalmente de 0,7 litros de dos de los productos (aceite de andiroba y copaiba), como indicador de la estabilidad de la demanda y de la venta. Mediante conversaciones con seis vendedores en diferentes momentos, se obtuvieron informaciones sobre los usos recomendados por ellos. De forma complementaria, se tuvieron en cuenta observaciones sobre prácticas de uso de la tierra en los alrededores de Santarém, realizadas durante giras de campo en el contexto de la ocupación principal del autor como asesor técnico del Servicio Alemán de Cooperación Técnica y Social, e informaciones obtenidas en conversaciones sostenidas con cinco informantes en comunidades rurales de la zona.

Es preciso señalar que la metodología aplicada tiene limitaciones inherentes en cuanto a la precisión y seguridad de la identificación botánica de las especies. Debido a que los productos que se venden en el mercado en su mayoría son aceites, resinas y fragmentos de cáscaras, generalmente carecen de características que permitan identificar la especie botánica de que provienen. De esta forma, en varios casos no ha sido posible determinar con seguridad la especie a través de comparaciones con descripciones científicas o muestras de herbario, ya que no se ha podido trazar toda la cadena productiva desde la recolección de la materia prima hasta la venta en el mercado. Además, no siempre los productos vendidos en el mercado con el mismo nombre, y utilizados de la misma forma, provienen de la misma especie botánica. En varios casos, pero no en todos, se han podido reducir las ambigüedades, preguntando a los vendedores sobre características fácilmente identificables como el tamaño de la planta, o sobre otras plantas con el mismo nombre. Siguiendo a Herrero Jáuregui et al. (2009), para hacer transparente el grado de ambigüedad se ha establecido una variable A (accuracy), la cual es explicada en la tabla 1, y a cada especie se ha asignado un valor de A.

Se tuvieron en cuenta los siguientes aspectos: las partes de las plantas empleadas para la obtención de los productos, los usos recomendados, los precios y sus cambios en el tiempo, y otros usos de las especies, principalmente para fines maderables. Se realizó una búsqueda de informaciones para confirmar la identidad taxonómica de las plantas de las que provienen los productos, así como sobre las 
características reproductivas y de crecimiento de las especies, sus mecanismos de dispersión, su hábitat preferido y sus densidades poblacionales en su hábitat natural. Con base en estas informaciones se analizaron diferentes aspectos de sostenibilidad del uso extractivo de los doce PFNM más vendidos.

En varios casos, para confirmar estadísticamente la asociación de determinadas características de las especies o de los productos, se ha aplicado el test exacto de Fisher (Fisher's Exact Probability Test) con 2 x 2 entradas, utilizando el software de acceso libre Vassarstats (http://vassarstats.net/ta2x2.html). Se trata de un test no paramétrico, adecuado para muestras pequeńas y datos en escala categorial.

\section{Resultados}

\section{Aspectos taxonómicos}

Doce productos fueron encontrados en por lo menos $90 \%$ de las visitas en el Mercadão 2000. Cada uno de estos productos proviene de una planta diferente. Las doce plantas pertenecen a doce diferentes géneros botánicos y, según se consideran las Caesalpiniaceae, Fabaceae y Leguminosae como tres familias o se fusionan en una sola, a diez o a ocho familias. Las fabáceas/leguminosas están representadas por tres o por cinco géneros respectivamente, y las nueve o siete familias restantes por un género cada una (tabla 1). En los casos de la andiroba y la copaiba, plantas cuyos nombres vernáculos se refieren a diferentes especies que se usan para la elaboración de los productos, se trata de especies que pertenecen a un solo género (andiroba - Carapa y copaiba - Copaifera).

\section{Usos curativos}

Los productos fueron recomendados por los vendedores para once diferentes usos curativos (tabla 2). Cuatro productos fueron recomendados para dolores musculares, tres para problemas de vías respiratorias y dos para diabetes, dolores de articulaciones y úlceras de estómago. Un solo producto se usa para cada una de las afectaciones ácido úrico alto, gastritis y colesterol alto, y también como cicatrizante, estimulante y afrodisíaco y como repelente de mosquitos. Un producto (resina de copaiba) fue recomendado para tres distintos usos y cinco productos (aceite de andiroba, aceite de piquiá, té de pau d'arco, té de sucupira y té de sacacá) para dos diferentes usos. Los seis productos restantes fueron recomendados para un solo uso. Hubo consistencia entre los usos que recomendaron los seis vendedores entrevistados, y el único caso en que un uso fue recomendado solamente por dos de ellos fue el uso del aceite de andiroba como repelente de mosquitos.

Tabla 1. Nombres comunes, especies y familias

\begin{tabular}{lllll}
\hline Nombre común & Nombre cientifico & A & Familia & Observaciones \\
\hline Amapá doce & Brosimum parinarioides Ducke & 1 & Moraceae & $\begin{array}{l}\text { Se distingue de Amapá } \\
\text { amargoso }\end{array}$ \\
\hline Andiroba & Carapa guianensis Aublet & 3 & Meliaceae & $\begin{array}{l}\text { Se utilizan las dos } \\
\text { especies }\end{array}$ \\
& o Carapa procera & & & \\
\hline Barbatimão & $\begin{array}{l}\text { Stryphnodendron adstringens } \\
\text { (Mart.) Coville }\end{array}$ & 3 & Mimosaceae & \\
\hline Catuaba & $\begin{array}{l}\text { Erythroxylum vaccinifolium } \\
\text { Mart. }\end{array}$ & 3 & Erythroxylaceae & $\begin{array}{l}\text { Arbusto hasta } \\
\text { Copaiba }\end{array}$ \\
\hline Cumarú & $\begin{array}{l}\text { Copaifera spp. } \\
\text { Dipteryx odorata (Aubl.) }\end{array}$ & 3 & Fabaceae & Se usan varias especies \\
\hline
\end{tabular}




\begin{tabular}{lllll}
\hline Nombre común & Nombre cientifico & A & Familia & Observaciones \\
\hline Ipê o & $\begin{array}{l}\text { Handroanthus impetiginosus } \\
\text { (Mart. ex D. C.) Mattos) o H. }\end{array}$ & 4 & Bignoniaceae & \\
Pau d'arco & serratifolius & & \\
\hline Jatobá (1) & Hymenaea courbaril L & 2 & Caesalpiniaceae \\
\hline Piquiá & Caryocar villosum (Aubl.) Pers. & 1 & Caryocaraceae \\
\hline Sacacá & Croton cajucara Benth. & 1 & Euphorbiaceae \\
\hline Sucupira & Bowdichia virgilioides Kunth. & 4 & Fabaceae \\
\hline Sucuuba & $\begin{array}{l}\text { Himatanthus sucuuba (Spruce) } \\
\text { Woods. }\end{array}$ & 1 & Apocynaceae \\
\hline
\end{tabular}

Fuente: elaboración propia

A corresponde a la variable accuracy en el trabajo de Herrero Jáuregui et al. (2009), que se refiere al grado de ambigüedad taxonómica de los nombres vernáculos. Los valores fueron tomados de la lista en aquel trabajo, a excepción de barbatimão,catuaba, sacacá y sucuuba, que no forman parte de la lista, y andiroba y copaiba, nombres que en el contexto del presente trabajo se refieren a dos o varias especies del mismo género, por lo que requieren una clasificación correspondiente. Significan: 1) un nombre vernáculo corresponde a una especie, 2) una especie tiene varios nombres vernáculos, 3) varias especies tienen el mismo nombre vernáculo, 4) la combinación de 2 y 3.

Los productos que se utilizan para dolores musculares y de articulaciones, y como cicatrizantes, son aceites y resinas, sean elaborados de las semillas, sean extraídos del tronco, mientras que los productos que se usan para otras afecciones en su gran mayoría son de otro tipo (té de semillas, té de cáscaras, tabla 2). Según el test exacto de probabilidades de Fisher (Fisher's Exact Probability Test), la asociación entre tipo de uso (dolores musculares y de articulaciones versus otros usos) y tipo de producto (aceite - té) es significativa, con una $\mathrm{p}=0,01$. Es preciso señalar que en ese análisis no se tuvo en cuenta el uso como repelente del aceite del aceite de andiroba, porque aparentemente no es su uso principal.

\section{Partes usadas de las plantas}

Cuatro de los doce productos $(33 \%$ del total) son semillas o productos elaborados a partir de semillas. En dos casos las semillas se utilizan directamente para la preparación de té (sucupira, Bowdichia virgilioides, y cumarú, Dipteryx odorata), y en dos casos se vende el aceite extraído artesanalmente de las semillas (andiroba, Carapa guianensis/C. procera, y piquiá, Caryocar villosum). Tres productos (25\% del total) son líquidos extraídos del tronco: la "leche" de amapá (Brosimum parinarioides), la "leche" de sucuuba (Himathanthus sucuuba) y la resina de copaiba (Copaifera spp.). Los cinco productos restantes ( $42 \%$ del total) son fragmentos de corteza que se usan para la preparación de té (sacacá, Croton cajucara; catuaba, Erythroxylum sp.; pau d'arco, Handroanthus impetiginosus; jatobá, Hymenaea courbaril; barbatimão, Stryphnodendron adstringens). Predomina el uso de productos elaborados a partir de partes vegetativas de los árboles (ocho de las doce especies, $67 \%$ del total), en relación con los productos de órganos reproductivos (cuatro de las doce especies, $33 \%$ del total). Si bien es cierto que la mayoría de los tés se preparan a partir de fragmentos de corteza, estadísticamente no hay asociación entre las formas de usos (aceite - té) y partes de las plantas (órganos generativos - órganos vegetativos), y el valor del $\mathrm{p}$ $(0,576$, Fisher's Exact Probabilitty Test) está lejos del nivel que indica una relación significativa entre ambas variables.

\section{Precios y volúmenes de ventas}

Una botella de aceite-resina de copaiba se estaba vendiendo por 22 reales al empezar las observaciones, y el precio subió a 24 reales hacia el final del período, correspondiendo en ese momento a 10 - 12 US\$ aproximadamente. No se observaron bajas de precio durante el tiempo de observación. En el mismo 
tiempo, la cantidad de mesas de venta aumentó de cinco a siete. No fue posible obtener informaciones fiables sobre el volumen de venta.

La cantidad de botellas presentes en la mesa varió entre 10 y 21 en el caso del aceite de andiroba, y entre 4 y 15 en el aceite de copaiba, con tendencia de aumentar. Los comentarios de uno de los vendedores indican que en el caso de las semillas de cumarú (Dipteryx odorata) el precio había experimentado un alza repentina en un momento del primer quinquenio de la década de 2000, y que después (por lo menos hasta principios de 2008) se mantenía en un mayor nivel, en comparación con el período anterior.

\section{Formas de vida y hábitat}

Diez de las doce plantas (83\%) cuyos productos son vendidos con regularidad en el Mercadão 2000 son árboles grandes que pueden alcanzar tamaños de mucho más de $10 \mathrm{~m}$, y cuyo hábitat son bosques naturales de tierra firme (tabla 3). Las dos especies restantes (Croton cajucara y Erythroxylum sp.) son árboles pequeños o arbustos cuyo tamaño generalmente no llega a $10 \mathrm{~m}$ (Conceição et al., 2002; Patricio y Pirani, 2002), y según tres informantes en las comunidades de la zona, su hábitat son bosques secundarios, matorrales y bordes de caminos (Croton), o el sotobosque de la vegetación natural (Erythroxylum). H. sucuuba (sucuuba) se encuentra no solamente en bosques de tierra firme, sino también en bosques de zonas inundables (Ferreiras et al., 2007) y en bosques secundarios. Bowdichia virgilioides (sucupira) no solamente está presente en bosques de tierra firme, sino también en bosques de cerradão en el estado de Maranhão, en la zona de transición del bosque amazónico hacia el cerrado (Conceição et al., 2011).

\section{Densidades de las poblaciones de las especies y áreas de distribución}

Para cinco de las doce especies (Caryocar villosum, Copaifera sp., Dipteryx odorata, Hymenaea courbaril, Handroanthus impetiginosus), los datos en Shanley y Medina (2005) y en Herrera Jáuregui et al. (2011a) indican densidades poblacionales muy bajas, de menos de 2 individuos por ha, en su hábitat natural. Para Carapa guianensis los datos de Shanley y Medina (2005) indican una densidad variable, que alcanza hasta 8 individuos/ha (tabla 3), y según Almeida et al. (2012), en un bosque cerca de Santarém, donde se había extraído madera, esta especie está presente en una densidad de 10,8 individuos/ha. Bowdichia virgilioides fue encontrada por Assunção y Felfili (2004) en una densidad de 4,0/ha en un fragmento de cerrado, cerca de Brasilia, y es una de las especies más frecuentes en la vegetación del cerradão en un lugar en el estado de Maranhão (Conceição et al., 2011). En cambio, en un bosque cerca de Santarém, estudiado por Almeida et al. (2012), Bowdichia no se encuentra entre las treinta especies de árboles con mayores índices de importancia (IVA).

Los datos disponibles para Stryphnodendron adstringens (Assunção y Felfili, 2004; Ortiz y Ribeiro, 2006) indican densidades sensiblemente mayores, de 10 individuos/ha y más (tabla 3). De todos modos, hay que tener en cuenta que estos estudios se realizaron fuera de la zona del bosque pluvial amazónico. Otro dato que indica bajas densidades de la mayoría de las especies de árboles estudiados aquí es que en el bosque mencionado de tierra firme en las cercanías de Santarém, aunque por lo menos siete de las especies estudiadas aquí estaban presentes, solamente una (Carapa guianensis) formaba parte de las treinta especies con mayores valores de importancia (Almeida et al., 2012). Hay que señalar que en ese bosque hubo extracción de madera.

De las referencias citadas en la tabla 3 para la mayoría de las especies estudiadas en el presente trabajo (Bowdichia, Carapa, Copaiba, Dipteryx, Handroanthus, Hymenea, Stryphnodendron), sus áreas de distribución se extienden por amplias áreas de Suramérica, en algunos casos en otro país (Leigue Gómez, 2011) o en otro bioma (Assunção y Felfili, 2004; Conceição et al., 2011; Ortiz y Ribeiro, 2006). En otras especies (Croton cajucara, Terceros et al., 2007; Costa y Mitja, 2010; Himathantus sucuuba, Alvino et al., 2005; Costa y Mitja, 2010), las referencias a usos tradicionales en otras zonas apuntan en el mismo sentido. 


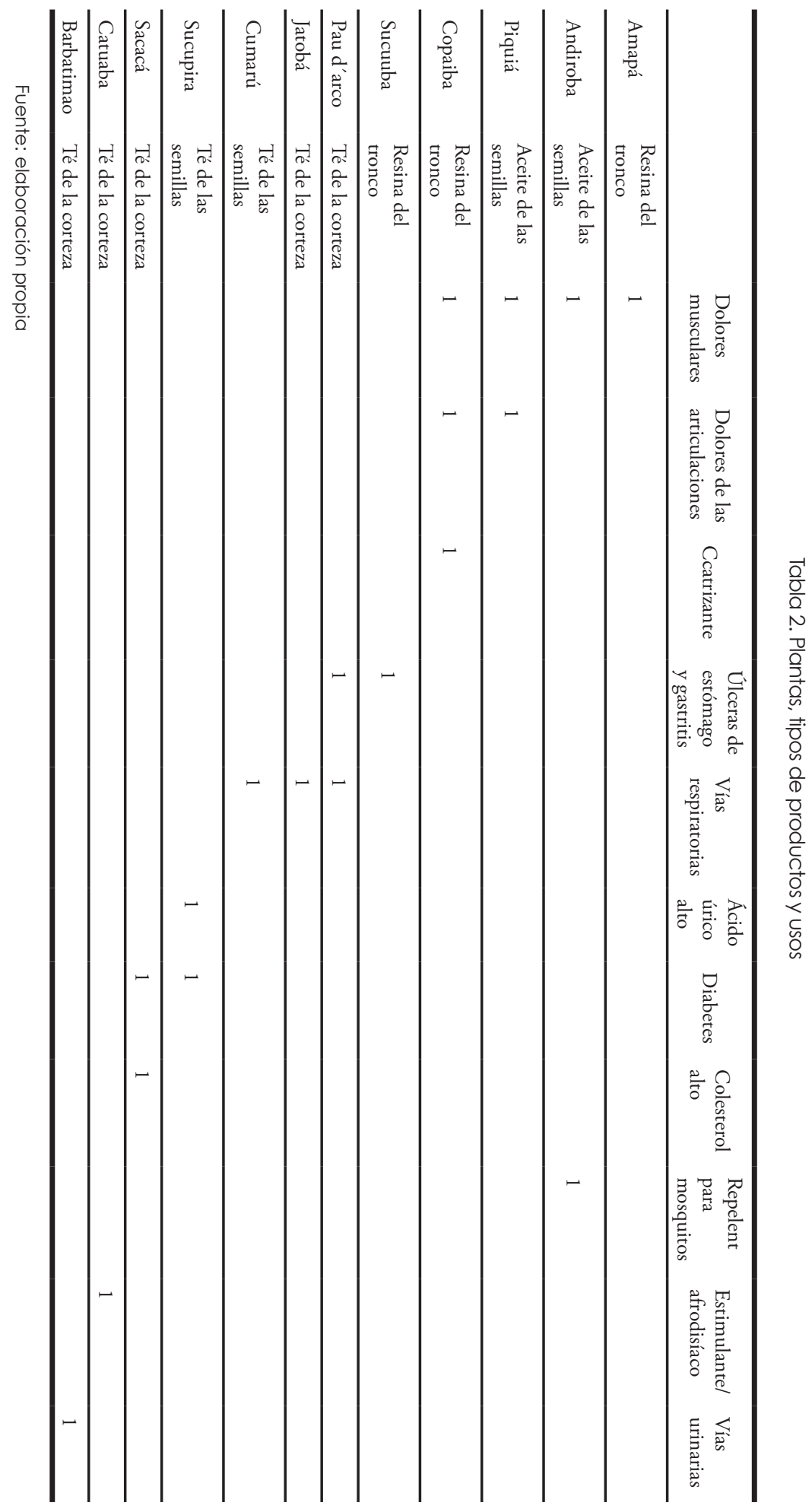


Tabla 3. Densidades en bosques naturales y uso maderero de las diez especies de árboles grandes

\begin{tabular}{lll}
\hline Nombre cientifico & $\begin{array}{l}\text { Densidad } \\
\text { (individuos/ha) }\end{array}$ & Uso maderero \\
\hline Brosimum parinarioides Ducke & & $\mathrm{x}(1,3)$ \\
\hline Carapa guianensis Aublet/C. procera & $1,0(2)-10,8(3)$ & $\mathrm{x}(1,3)$ \\
\hline Stryphnodendron adstringens (Mart.) Coville & $10,2(6)-98(7)$ & \\
\hline Copaifera langsdorffi (Desf.) Kuntze/C. reticulata Ducke & $0,1-2,0(2), 0,8(6)$ & $\mathrm{x}(1)$ \\
\hline Dipteryx odorata (Aubl.) Willd. & $0,2(4)-5,4(5)$ & $\mathrm{x}(1)$ \\
\hline Handroanthus impetiginosus (Mart. ex D. C.) & $0,1-0,3(5)$ & $\mathrm{x}(1)$ \\
\hline Hymenaea courbaril L & $0,2-2(3)$, & $\mathrm{x}(1)$ \\
\hline Caryocar villosum (Aubl.) Pers. & $0,4-0,6(2)$ & $\mathrm{x}(1,3)$ \\
\hline Bowdichia virgilioides Kunth. & $0,6(6)-4,0(7)$ & $\mathrm{x}(1,3)$ \\
\hline Himatanthus sucuuba (Spruce) Woods. & & \\
\hline
\end{tabular}

Fuentes de los datos:

(1) Herrero Jáuregui et al. (2009)

(5) Shanley et al. (2012)

(2) Shanley y Medina (2005)

(6) Ortiz y Ribeiro (2006)

(3) Almeida et al. (2012)

(7) Assuncao y Felfili (2004)

(4) Herrero Jáuregui et al. (2011 b)

\section{Características de las semillas y agentes de dispersión}

Las semillas de ocho de las doce especies ( $67 \%$ del total) son dispersadas por animales, principalmente mamíferos y aves. Entre estas especies se encuentran tres cuyas semillas son usadas para fines curativas (Carapa guianensis, Caryocar villosum y Dipteryx odorata). Para cinco de las ocho especies dispersadas por animales hay constancia de que sus frutos caídos en el suelo o sus flores caídas atraen animales de caza (tabla 4, Shanley y Medina, 2005; Herrero Jáuregui et al., 2011b). Las cuatro especies restantes son dispersadas por el viento o son autócoras, y en una de ellas, que se encuentra no solamente en tierra firme sino también en la varzea (Himathantus sucuuba), el agua también es un agente dispersador. No hay asociación entre la forma de dispersión (zoocoría - otras formas) y las partes de las plantas usadas (partes reproductivas - partes vegetativas), con un valor de $\mathrm{p}=0,594$, aplicando el Test Exacto de Fisher.

Tabla 4. Agentes de dispersión, atracción de animales de caza y hábitat

\begin{tabular}{llll}
\hline Especie & Agente de dispersión & Atrae animales de caza & Hábitat \\
\hline Brosimum parinarioides Ducke & Zoo (2) & - & BTF \\
\hline Carapa guianensis, C. procera & Zoo (3) & Sí (frutos) 2) & BTF, BG \\
\hline $\begin{array}{l}\text { Stryphnodendron adstringens (Mart.) } \\
\text { Coville }\end{array}$ & Zoo (9) & - & BTF \\
\hline $\begin{array}{l}\text { Erythroxylum vaccinifolium Mart. } \\
1940\end{array}$ & Zoo (6) & - & BTF-SB \\
\hline Copaifera langsdorffi, C. reticulata & Zoo (4) & Sí (frutos) (3) & BTF \\
\hline Dipteryx odorata & Zoo (3, 4) & Sí (frutos) (3) & BTF \\
\hline
\end{tabular}




\begin{tabular}{llll}
\hline Especie & Agente de dispersión & Atrae animales de caza & Hábitat \\
\hline Handroanthus impetiginosus & Ane $(1)$ & - & BTF \\
\hline Hymenaea courbaril L. & Zoo $(3)$ & Sí (frutos) $(4)$ & BTF \\
\hline Croton cajucara Benth. & Aut $(5)$ & - & BTF \\
\hline Caryocar villosum (Aubl.) Pers. & Zoo $(6)$ & Sí (frutos y flores) (4) & BTF \\
\hline Bowdichia virgilioides Kunth & Ane $(1)$ & - & BTF, CE \\
\hline Himathanthus sucuuba & Ane $(7,8$,$) (agua!)$ & - & BTF, BS, vZ \\
\hline
\end{tabular}

Fuentes de los datos:

(1) Conceição et al. (2011)

(2) Gandtner y Chevrette (2013)

(3) Shanley y Medina (2005)

(4) Herrero Jáuregui et al. (201 1a)

(5) Conceição et al. (2002) de sotobosque, vz: bosque inundable (varzea)

(6) Patricio y Pirani (2002)

(7) Ferreiras et al. (2007)

(8) Wittmann et al. (2006)

(9) Felfili et al. (1999)

La asociación entre la forma de dispersión y la relación de la especie con animales de caza (atracción - no hay constancia de atracción) no es significativa, pero el valor de p en el Test Exacto de Fisher $(0,070)$ confirma que existe una tendencia a que las semillas zoocoras atraigan animales de caza.

\section{Uso de la madera de las especies}

De las diez especies arbóreas de gran tamaño cuyos productos fueron encontrados con regularidad en el Mercadão 2000, cinco son utilizadas para madera en la zona (Caryocar villosum, Copaiba reticulata, Dipteryx odorata, Hymenea courbaril y Handroanthus impetiginosus). Tres más (Bowdichia virgilioides, Brosimum parinarioides, Carapa guianensis/C. procera) se usan para madera en otras zonas del estado de Pará (Herrero Jáureguiet al., 2009).

Entre las diez especies de árboles grandes, solamente para Himathanthus sucuuba y Stryphnodendron adstringens no se encontraron referencias a un uso comercial de la madera. Para las dos especies, Alvino et al. (2005) de la zona bragantina en el este de Pará solo mencionan usos de PFNM. Esto significa que $67 \%$ de las especies estudiadas aquí y $80 \%$ de las especies de árboles son de uso maderero. Según informaciones recogidas de los vendedores, en varias especies (Brosimum parinariodes, Copaifera langsdorfii, Hymenaea courbaril y Handroanthus impetiginosus) de las que se usa la corteza o la resina con fines curativos, estos productos también se aprovechan de los troncos después de talar los árboles, si bien el objetivo principal del corte es el aserrío de la madera.

\section{Plantaciones de árboles de productos no maderables}

Las especies cumarú (Dipteryx odorata), pau-d'arco (Handroanthus impetiginosus.), andiroba (Carapa spp.) y jatobá (Hymenaea courbaril) son utilizadas en la zona como componentes arbóreas de sistemas agroforestales (May, 2013). Handroanthus —en aquel trabajo referido con su nombre antiguo, Tabaebuia impetiginosa - ha sido plantado también de forma pura. Mientras que en el caso de Handroanthus el objetivo económico primordial de las plantaciones es la producción de madera, en el caso de los sistemas agroforestales con Carapa y Dipteryx, para los productores familiares la producción de semillas para usos medicinales o industriales es un objetivo importante. Esto no excluye que la perspectiva de un futuro uso de la madera también haya motivado su plantación.

En una aldea de la Floresta Nacional de Tapajós, al sur de Santarém, donde se estaba desarrollando un microproyecto de cooperación internacional de extracción y comercialización de aceites y otros 
productos no maderables del bosque (Araujo, 2002), pequeñas plantaciones de copaiba (Copaifera sp.) y andiroba (Carapa guianensis) habían sido establecidas por un comunitario, por iniciativa propia, en terrenos en barbecho. Mientras la plantación de copaiba en 2007 a los dos años fue abandonada, porque el que la había iniciado consideraba el crecimiento demasiado lento, el mismo comunitario veía como promisoria la plantación de andiroba, por su crecimiento más rápido. Otras personas entrevistadas de la misma aldea compartieron ese criterio.

\section{Discusión}

\section{Impactos externos en la capacidad de los sistemas de generar los recursos}

$\mathrm{Al}$ igual que en la región de Belém, en el este de Pará, la mayor parte de los productos más vendidos en Santarém provienen de árboles de bosques primarios. Con $83 \%$ de árboles grandes y $80 \%$ de ellos de interés maderero, actual o potencial, la predominancia de árboles como fuente de los PFNM en el Mercadão 2000 de Santarém era aun más grande que en Belém (Shanley y Luz, 2003). Esto indica que la extracción de madera, al igual que la conversión de bosques en terrenos de uso agropecuario, tiene el potencial de poner en peligro la base de recursos para el uso de los PFNM.

Irónicamente, a corto plazo el uso maderero contribuye a aumentar la oferta de estos productos. Según informaciones de los vendedores en el Mercadão 2000, el aprovechamiento de la corteza de árboles de Hymenaea y Handroanthus que fueron cortados para madera es práctica común, así como el aprovechamiento de la resina de los troncos cortados de Copaifera. Según Shanley y Medina (2005, p. 101), en Pará oriental, la corteza de Handroanthus impetiginosus es aprovechada en los aserraderos, y en algunos casos es comercializada por los madereros. Veiga Junior y Pinto (2002) afirman que en ciertas partes de la Amazonía el aceite de copaiba se ha convertido en un producto secundario del corte de madera.

De todos modos, es previsible que a largo plazo la tendencia sea diferente. Diez años después de un período de extracción intensa de madera en los bosques en los alrededores de una comunidad de la Amazonía oriental, la disponibilidad de los PFNM provenientes de Dipteryx odorata, al igual que de Caryocar villosum, Copaifera spp., Carapa guianensis y Hymenaea courbaril experimentó descensos de 63 a $80 \%$, según la especie (Rist et al., 2011). En aquel estudio no solamente se tuvo en cuenta el uso de las especies para productos comerciables o de uso casero, sino también servicios como la atracción de animales de caza por las semillas y flores caídas al suelo. Todas las cinco especies mencionadas forman parte de los árboles que son fuente de los PFNM de uso curativo más vendidos en Santarém, y tienen la función de atraer a los animales de caza (tabla 4), aspecto importante para el autoconsumo de las comunidades. Además, tres de las cuatro especies que según Herrero Jáuregui et al. (2009) cuentan con el mayor riesgo de conflicto entre el uso maderable y el no maderable (Dipteryx odorata, Handroanthus impetiginosus e Hymenaea courbaril) forman parte de las especies de las que se elaboran los productos de uso curativo más vendidos en el Mercadão 2000.

\section{Impactos externos en los aspectos económicos del sistema}

El mayor precio del aceite-resina de copaiba en el Mercadão 2000 de Santarém durante los años 2006 a 2008, en comparación con los precios del mismo producto en el año 2000 en el mercado Ver-o-peso de Belém (Shanley y Luz 2003), probablemente refleja, sobre todo, la tendencia de un ascenso paulatino de los precios locales de los productos naturales curativos que mencionan estas autoras. La ampliación del uso de sacacá (Croton cajucara) hacia el sur y sureste de Brasil, en años recientes (Rodríguez et al., 2010), se corresponde bien con esta tendencia de una mayor demanda, con el consiguiente aumento del precio. La comparación de los niveles de precios en Santarém y Belém también indica una relativa homogeneidad regional, y confirma el hecho de que los cambios de precios locales para consumidores de estos productos tienden a ser suaves, y no abruptos. De todas maneras, la estabilidad relativa de los precios locales que fue observada en los aceites de andiroba y copaiba no significa que no se puedan hacer sentir cambios en el mercado internacional. Tampoco significa necesariamente que los precios 
pagados a las personas que recolectan la materia prima y elaboran los productos sean de una estabilidad relativa parecida. Para evaluar la influencia de posibles cambios en la demanda internacional sería necesario observar los precios y los volúmenes de venta durante un período de observación más largo.

\section{Impactos internos en la capacidad de los sistemas de generación de los recursos}

Un factor interno que afecta negativamente la sostenibilidad del sistema son las importantes fluctuaciones interanuales naturales de la producción de semillas que son mencionadas por Shanley et al. (2002), y que también describe Arteaga (2007) con relación a los bosques tropicales de Bolivia. De estas fluctuaciones en la oferta local hay que esperar repercusiones en la constancia de la oferta y en los precios.

En lo que se refiere a la cuestión de si las mismas prácticas de uso extractivas pueden tener impactos negativos en la disponibilidad del recurso, es preciso evaluar los productos de forma diferenciada y tener en cuenta las características ecológicas de las especies a partir de las que se elaboran. En el caso de la resina de copaiba, las técnicas tradicionales de aprovechamiento, haciendo cortes con hachas en la base del tronco (Plowden, 2003) eran claramente destructivas. Sin embargo, se han desarrollado técnicas de perforación con taladros que no afectan a los árboles (Araujo, 2002), que se estaban practicando con éxito en la Floresta Nacional de Tapajós durante el período de estudio del presente trabajo. Shanley y Medina (2005) citan investigaciones cuyos resultados preliminares indican que la corteza del pau d'arco (Handroanthus impetiginosus) tiene una buena capacidad de regeneración y se puede usar de forma repetida sin hacerle daño al árbol, cuando no se le anilla y se mantiene el flujo de savia. Sin embargo, en otras especies como el jatobá (Hymena courbaril), hay evidencias de que la capacidad de regeneración de la corteza es más débil (Shanley y Medina, 2005).

El uso de frutos y semillas, aunque a corto plazo no afecta a las poblaciones de los árboles, a mediano y largo plazo puede afectar la regeneración natural y el mantenimiento de las poblaciones, como indican los resultados de Peres et al. (2003) para la castańa (Bertholletia excelsa). Con respecto a una de las especies estudiadas aquí (cumarú, Dipteryx odorata), según Herrero Jáuregui et al. (2011a) no se han observado impactos negativos sensibles con una extracción de las semillas de intensidad no excesivamente fuerte, a pesar de que esta especie tiene características reproductivas (bajas distancias de dispersión y alta mortalidad de plántulas silvestres) que hacen suponer que sea vulnerable a la recolección de sus semillas. Según Forget y Jansen (2006), la extracción de semillas de Carapa procera que no son dispersadas, probablemente no afectaría sensiblemente la regeneración de la población de los árboles. De esta manera, es posible que en el caso de las tres especies estudiadas aquí, cuyas semillas son utilizadas para la producción de aceite y son dispersadas por mamíferos (Carapa spp., Caryocar villosum y Dipteryx odorata), la recogida de las semillas para la elaboración de los productos no maderables de uso curativo tenga poco impacto en su regeneración natural. En síntesis, se necesitan más estudios específicos para evaluar posibles efectos negativos de la extracción de productos no maderables en la disponibilidad del recurso.

\section{Aspectos económicos y sociales internos}

Como limitante para el uso de productos forestales no maderables con fines comerciales, Shanley et al. (2002) mencionan los problemas crónicos de transportación y la poca experiencia de los usuarios en la comercialización. Conversaciones con personas en comunidades en los alrededores de Santarém confirmaron que este también es el caso en la zona.

Tal como afirman Shanley y Medina (2005), en poblaciones silvestres de poca densidad, como es el caso de las especies estudiadas aquí, el trabajo necesario para la cosecha y el acopio generalmente es considerable. Este aspecto adquiere aun más importancia cuando las poblaciones de árboles relativamente cercanas a los asentamientos humanos se vuelen escasas, por cambios en el uso de la tierra o por explotación maderera de los bosques, y los lugares de recolección se encuentran a distancias cada vez mayores. Existen pues razones prácticas para el establecimiento de plantaciones de árboles cuyos productos se utilizan, como la mayor concentración del recurso en el espacio, lo que reduce el 
tiempo de trabajo necesario para la cosecha y el acopio. En este sentido, como lo afirman Shanley et al. (2002), para lugares relativamente próximos a centros urbanos, el establecimiento de plantaciones de árboles con fines de producción no maderable cerca de los asentamientos humanos, o también el enriquecimiento del bosque con determinadas especies de interés podría ser una estrategia de adaptación a la disminución de la accesibilidad al recurso.

En el caso de la andiroba (C. guianensis/C. procera), el manejo de la reproducción es fácil (Ferraz et al., 2002) y el crecimiento es relativamente rápido. Tonini et al. (2005), en una plantación de esa especie en el estado brasileño de Roraima, encontraron tasas medias anuales de crecimiento de $1,3 \mathrm{~m}$ por año en altura y $6,3 \mathrm{~m}$ en volumen durante los primeros siete años. La edad de madurez sexual es relativamente baja, y la especie ha sido utilizada en plantaciones agroforestales en la región por agricultores familiares (May, 2013). Un objetivo importante en ese caso ha sido la producción de semillas para aceite. La situación es parecida en el caso del cumarú (Marques y Ferreira, 1995; May, 2013). En el caso de Hymenea courbaril, también se observa cierto interés de parte de algunos agricultores familiares por hacer plantaciones en los alrededores de Santarém, aunque el crecimiento es sensiblemente más lento, con 0,8 y $2,9 \mathrm{~m} / \mathrm{a}$ respectivamente, según Tonini et al. (2005). En cambio, en el caso de la copaiba (Copaifera sp.), las perspectivas de poder aprovechar productos no maderables de plantaciones en un plazo razonable son menos promisorias, ya que los árboles con diámetros menores de $45 \mathrm{~cm}$ solamente producen cantidades insignificantes de resina (Plowden, 2003).

\section{Conclusiones}

Los resultados indican que la mayor posibilidad de poner en peligro la sostenibilidad del uso de productos no maderables forestales en los alrededores proviene de factores externos, específicamente los cambios del uso de la tierra y el uso no sostenible de la madera. En vista de esa situación, se requiere un manejo múltiple de los bosques, como lo recomiendan Guariguata et al. $(2009,2010)$, teniendo en cuenta igualmente el uso maderable y los diferentes usos no maderables de los bosques, incluyendo servicios ecológicos y de subsistencia de los moradores locales.

Con respecto a los factores internos de los sistemas de generar los recursos, se observan potenciales de uso sostenibles, si bien es cierto que varían de una especie a otra. La reducida experiencia en la comercialización de los PFNM en mercados distantes es una limitante, cuya incidencia probablemente es menor en los mercados locales. Los dos aspectos — resiliencia de los sistemas de generación de recursos y estabilidad de la comercialización local— requieren más investigaciones.

La plantación de especies de árboles con el objetivo de usar sus productos no maderables, en varios casos se vislumbra como una alternativa promisoria, con el potencial de reducir el tiempo de trabajo necesario para recolección y acopio. Ciertamente, al utilizar los productos de plantaciones de árboles, en lugar de los productos no maderables de bosques naturales, es de esperar que se tienda a reducir la vinculación de los productores con estos ecosistemas naturales y su interés económico en la conservación de estos recursos.

Agradecimientos: el autor agradece a todos los involucrados por su colaboración, específicamente a los vendedores de los PFNM, a los productores campesinos entrevistados, así como a dos revisores anónimos por sus valiosas sugerencias. 


\section{Referencias}

Almeida, L. S., Gama, J. R. V., Oliveira, F. A., Carvalho, O. P., Gonçalves, D. C. M. y Araújo, G. C. (2012). Fitossociologia e uso múltiplo de especies arbóreas em floresta manejada, comunidade Santo Antonio, municipio de Santarém, estado do Pará. Acta Amazônica, 42 (2), 185-194.

Almeida, M. W. B. (2004). Direitos á floresta e ambientalismo: seringueiros e suas lutas. Revista Brasileira de Ciencias Sociais, 19 (55), 33-52.

Alvino, F. O., Silva, M. F. F. y Rayol, B. P. (2005). Potential de uso das espécies arbóreas de uma floresta secundaria, na zona Bragantina, Pará, Brasil. Acta Amazonica, 35 (4), 413-420.

Araujo, V. G. (2002). Floresta Nacional do Tapajós. Levantamento de mercado de produtos florestais não madeireiros. Santarém (Pará): ProManejo, projeto de apoio ao manejo florestal sustentável na Amazônia.

Arnés Prieto, E. (2011). Desarrollo de la metodología de la evaluación de la sostenibilidad de los campesinos de montaña en San José de Cusmapa (Nicaragua) (tesis de maestría). Universidad Politécnica de Madrid.

Arteaga, L. L. (2007). Fenología y producción de semillas de especies arbóreas maderables en un bosque húmedo montano de Bolivia (pN Anmi Cotapata). Revista Boliviana de Ecología y Conservación Ambiental, 21, 57-68.

Assunção, S. L y Felfili, J. M. (2004). Fitossociologia de um fragmento de cerrado sensu stricto na APA do Paranoá, DF, Brasil. Acta Botanica Brasilica, 18 (4), 903-909.

Bacha, C. J. C. y Rodríguez, L. C. E. (2005). The people of Tapajós. ITTO Tropical Forest Update, 15 (4), 10-11.

Becker, C. D. y Ostrom, E. (1995). Human ecology and resource sustainability: the importance of institutional diversity. Annual Review for Ecology and Systematics, 26, 113-133.

Carpenter, S. R., Mooney, H. A., Agard, J., Capistrano, D., DeFries, R. S., Díaz, S., Dietz, T., Duraiappah, A. K., Oteng-Yeboah, A., Pereira, H. M., Perrings, C., Reid, W. V., Sarukhan, J., Scholes, R. J. y Whyteo, A. (2009). Science formanaging ecosystem services: beyond the Millenium Ecosystem Assessment. Proceedings of the National Academy of Sciences, 106 (5), 1305-1312.

Carrasco, P. (2005). Manejo del jebe (Hevea brasiliensis) en la Provincia de Tahuamanu - Madre de Dios. Iquitos (Perú): IIAP - Biodamaz.

Conceição, C. C. C.,Motta, G. C., Nascimento, M. E. y Vieira, I. M. S. (2002). Morfología das flores, frutos, sementes e plántulas de sacacá - Croton cajucara Benth. (Euphorbiaceae). Acta Horticulturae, 167-171.

Conceição, G. M., Ruggieri, A. C., Silva, E. O, Gomes, E. C. y Roche, A. M. V. (2011). Especies vegetales y síndromes de dispersión del área de protección ambiental municipal de Inhamum, Caixas, Maranhão, Brasil. Ambiente \& Agua, 6 (2), 129-142.

Costa, J. R. y Mitja, D. (2010). Uso dos recursos vegetais por agricultores familiares de Manacapuru (AM). Acta Amazonica 40 (1), 49-58.

De Groot, J. I. M. y Steg, L. (2009). Mean or green: which values can promote stable pro-environmental behavior? Conservation Letters, 2, 161-166.

Felfili, J. M., Junior, M. C. S., Dias, B. J. y Rezende, A. V. (1999). Estudo fenológico de Stryphnodendron adstringens (Mart.) Coville no cerrado sensu stricto da Fazenda Agua Limpa no Distrito Federal, Brasil. Revista Brasileira de Botânica, 22 (1), 83-90.

Ferraz, Isolde D. K., Camargo, J. L. C. y Sampaio, Paulo de T. B. (2002). Sementes e plántulas de andiroba (Carapa guianensis Aubl. e Carapa procera D. C.): aspectos botânicos, ecológicos e tecnológicos. Acta Amazônica, 32 (4), 647-661. 
Ferreiras, C. S., Piedade, M. T. F., Junk, W. J. y Parolin, P. (2007). Floodplain and upland populations of Amazonian Himatanthus sucuuba: Effects of flooding on germination, seedling growth and mortality. Environmental and Experimental Botany, 60 (3), 477-483.

Forget, P. M. y Jansen, P. A. (2006). Hunting increases dispersal limitation in the tree Carapa procera, a nontimber forest product. Conservation Biology, 21, 106-113.

Gandtner, M. M. y Chevrette, J. (2013). Dictionary of trees. Volume 2: South America: Nomenclature, taxonomy and ecology. Laval University, Quebec, Canadá.

Guariguata, M. R., García-Fernández, C., Nasi, R., Sheil, D., Herrero-Jáuregui, C., Cronkleton, P., Ndoye, O. e Ingram, V. (2009). Hacia un manejo múltiple en bosques tropicales. Consideraciones sobre la compatibilidad del manejo de madera y productos forestales no maderables. Cifor, Bogor, Indonesia.

Guariguata, M. R., Garcia-Fernandez, C., Sheil, D., Nasi, R., Herrero-Jáuregui, C., Cronkleton, P. e Ingram, V. (2010). Compatibility of timber and non-timber forest product management in natural tropical forests: perspectives, challenges, and opportunities. Forest Ecology and Management, 259 (3), 237-245.

Herrero Jáuregui, C., García Fernández, C., Sist, P. L. J. y Casado, M. A. (2009). Conflict of use for multi-purpose tree species in the state of Pará, eastern Amazonia, Brasil. Biodiversity and Conservation, 18 (6), 1019-1044.

Herrero Jáuregui, C., García Fernández, C., Sist, P. L. J. y Casado, M. A. (2011a). Recruitment dynamics of two low density neotropical multiple use tree species. Plant Ecology, 212 (9), 1501-1512.

Herrero Jáuregui, C., Pokorny, B. y Casado, M. A. (2011b). Coming down to earth: a critical analysis of a project for the commercialization of non-timber forest products in a community of the Eastern Amazon. Brazilian Journal of Forestry Research, 31 (66), 131-142

Holling, C. S. (1973). Resilience and stability of ecological systems. Annual Review of Ecology and Systematics, 4, 1-23.

Leigue Gómez, J. W. (2011). Regeneración natural de nueve especies maderables en un bosque intervenido de la Amazonía boliviana. Acta Amazónica, 41 (1), 135-142.

López Camacho, R. (2008). Productos forestales no maderables: importancia e impacto de su aprovechamiento. Revista Colombia Forestal, 11, 215-231.

Marques, L. C. T. y Ferreira, C. A. P. (1995). An agroforestry system in an area altered by shifting agriculture along the Santarém-Cuiaba highway, Para - Brasil. En J. A. Parotta y M. Kanashiro (Eds.), Management and rehabilitation of degraded lands and secondary forests in Amazonia. Proceedings of an international workshop, Santarem, Pará, Brasil, 18 - 22 April, 1993 (pp. 22-32). Santarem.

Masera, O., Astier, M. y López-Ridaura, S. (2000). Sustentabilidad y manejo de recursos naturales, el marco de evaluación Mesmis. México: Mundi-Prensa.

May, T. (2013). Sistemas agroforestales de colonos como alternativa de uso ecológicamente sustentable en el oeste de Pará, Brasil. Adopción y propuestas para su desarrollo. Ambiente y Desarrollo, 17 (32), 67-78.

Ortiz, L. F. y Ribeiro, C. H. R. A. (2006). Evaluación de parámetros geoecológicos para establecer correlaciones entre la vegetación y los suelos en la cuenca del río Chiqueiro, Municipio de Gouveia, Minas Gerais (Brasil). Revista Colombia Forestal, 9 (19), 155-176.

Patrício, M. C. B. y Pirani, J. R. (2002). Flora da Serra do Cipó, Minas Gerais: Erythroxylaceae. Boletim de Botánica, Departamento de Botánica, Instituto de Biociencias, Universidade de São Paulo, 20, 53-61.

Peres, C. A., Baider, C., Zuidema, P. A., Wadt, L. H. O., Kainer, K. A., Gomes-Silva, D. A. P., Salomão, R. P., Simoes, L. L., Franciosi, E. R. N., Cornejo Valverde, F., Gribel, R., Shepard Jr., G. H., Kanashiro, M., 
Coventry, P., Douglas, W. Y., Watkinson, A. R. y Freckleton, R. P. (2003). Demographic threats to the sustainability of Brazl nut exploitation. Science 302 (5653), 2112-2114.

Plowden, C. (2003). Production ecology of Coipaiba (Copaifera sp.) in the Eastern Brazilian Amazon. Journal of Economic Botany, 57 (4), 491-501.

Rist, L., Shanley, P., Sunderland, T., Sheil, D., Ndoye, O., Liswanti, N. y Tieguhong, J. (2012). The impacts of selective logging on non-timber forest products of livelihood importance. Forest Ecology and Management, 268, 57-69.

Rodrigues, G., Marcolín, E., Bona, S., Porawski, M., Lehmann, M. y Marroni, N. P. (2010). Hepatics alterations and genotoxic effects of Croton cajucara Benth. (sacacá) in diabetic rats. Arquivos de Gastroenterologia, 47 (3), 301-305.

Shanley, P. y Luz, L. (2003). The impacts of forest degradation on medicinal plant use and implications for health care in Eastern Amazonia. BioScience, 53 (6), 573-584.

Shanley, P., Luz, L. y Swingland, I. R. (2002). The faint promise of a distant market: a survey of Belem's trade in non-timber forest products. Biodiversity and Conservation, 11, 615-636.

Shanley, P. y Medina, G. (2005). Frutiferas e plantas úteis na vida amazônica. Belém/Pará: Cifor/Imazon.

Shanley, P., Silva, M. da S., Melo, T., Carmenta, R. y Nasi, R. (2012). From conflict of use to multiple use: Forest management innovations by smallholders in Amazonian logging frontiers. Forest Ecology and Management, 268, 70-80.

Terceros, P., Quelca, B. y Solares, M. (2007). Plantas medicinales en Bolivia. Estado de arte. La Paz, Bolivia: Organización de las Naciones Unidas para el Desarrollo Industrial y Gobierno de Bolivia, Ministerio de Planificación y del Desarrollo, Viceministro de Ciencia y Tecnología.

Tonini, H., Arco-Verde, M. F. y Pereira de Sá, S. (2005). Dendrometria de espécies nativas em plantios homogêneos no estado de Roraima - andiroba (Carapa guianensis Aubl.), Castanho-do Brasil (Bertholletia excelsa Bompl.), ipê-roxo (Tabaebuia avellanedea Lorentz ex. Griseb.) e jatobá (Hymenaea courbaril L.). Acta Amazônica, 35 (3), 353-362.

Veiga Junior, W. F. y Pinto, A. C. (2002). O gênero Copaifera L. Química Nova (São Paulo), 25 (2), 273-286.

Weinstein, B. (1997). Penetración del capital y problemas de control de la mano de obra en el comercio amazónico del caucho. Memoria y Sociedad, 2 (3) (Universidad Javeriana, Bogotá), 57-73.

Wittmann, F., Schöngart, J. y Montero, J. C. (2006). Tree species composition and diversity gradients in white-water forests across the Amazon Basin. Journal of Biogeography, 33, 1334-1347.

Young, T. y Burton, M. P. (1992). Agricultural sustainability: definition and implications for agricultural and trade policy. FaO Economic and Social Development Paper 110. Roma, FaO.

Zuidema, P. A. (2003). Ecología y manejo del árbol de castańa (Bertholletia excelsa). Riberalta (Beni, Bolivia) y Utrecht (Holanda): Promab (Universidad Técnica de Beni e Universidad de Utrecht). 